\title{
Local polynomial estimations of time-varying coefficients for local stationary diffusion models
}

\author{
Jixia Wang ${ }^{1,2^{*}}$ and Qingxian Xiao ${ }^{1}$
}

\section{"Correspondence:}

jixiawang@163.com

${ }^{1}$ Business School, University of

Shanghai for Science and

Technology, Shanghai, 200093,

China

${ }^{2}$ College of Mathematics and Information Science, Henan Normal University, Xinxiang, Henan 453007, China

\begin{abstract}
This paper is dedicated to the study of local polynomial estimations of time-varying coefficients for a local stationary diffusion model. Based on local polynomial fitting, the estimations of drift parametric functions are obtained by using local weighted least squares method. By applying the forward Kolmogorov equation, the estimation of the diffusion coefficient is proposed. The consistency, asymptotic normality and uniform convergence of the estimations that we proposed are established.
\end{abstract}

Keywords: local stationary model; local polynomial fitting; consistency; asymptotic normality; convergence rate

\section{Introduction}

The theory of local stationary process is introduced by Dahlhaus $[1,2]$. Intuitively speaking, a process is locally stationary if the process behaves like a stationary diffusion process in a neighborhood of a chosen time point. In recent years, various efforts have been made to explicitly express the local stationary models. For example, Koo and Linton [3] discuss semi-parametric estimations of a class of locally stationary diffusion processes with locally linear drift. Vogt [4] considers nonparametric regression for a locally stationary time series model. To the best of our knowledge, few literature works have researched local polynomial estimations in locally stationary time-inhomogeneous diffusion models. This motivates us to consider the local estimations of the time-varying coefficients for locally stationary diffusion models.

In this paper, we study local polynomial estimations of time-varying coefficients for local stationary diffusion models. Firstly, we propose the local estimations of drift time-varying parameters by using the local weighted least squares method. The techniques that we employ here are based on local polynomial fitting (see Fan and Gijbels [5]). Secondly, by using the forward Kolmogorov equation, we obtain the estimation of the diffusion coefficient which is an unknown function of both time and state. Finally, we establish the consistency, asymptotic normality and uniform convergence of our proposed estimations.

The remainder of this paper is organized as follows. In Section 2, we introduce our diffusion models and give the definition of the local stationarity. In Section 3, the local polynomial estimations of the drift parameters are proposed, and the consistency, asymptotic normality and uniform convergence rate of the estimations for the drift functions are established. In Section 4, we give the estimation of diffusion coefficient and verify its

(O2014 Wang and Xiao; licensee Springer. This is an Open Access article distributed under the terms of the Creative Commons Attribution License (http://creativecommons.org/licenses/by/2.0), which permits unrestricted use, distribution, and reproduction in any medium, provided the original work is properly cited. 
asymptotic properties. Proofs of the main results are given in Section 5. In Section 6, the conclusions of this paper are given.

\section{Methods and local stationary}

Suppose that the diffusion process $\left\{X_{t, T}, t, T \geq 0\right\}$ satisfies

$$
d X_{t, T}=\left[\alpha(t / T)+\beta(t / T) g\left(X_{t, T}\right)\right] d t+\sigma\left(t / T, X_{t, T}\right) d B_{t}, \quad X_{0, T}=x_{0},
$$

where $g(\cdot)$ is a known Borel function, and $\left\{B_{t}: t \geq 0\right\}$ is a standard Brownian motion defined on the filtered probability space $\left(\Omega, F^{B},\left(F_{t}^{B}\right), P\right)$, where $F^{B}$ is a $\sigma$-algebra, $F_{t}^{B}$ is a filtration. The stochastic process $\left\{X_{t, T}\right\}$ defined as the solution to model (1) has a domain $I=[l, r]$, where $-\infty \leq l<r \leq+\infty$. Here, $x_{0}$ is a given random variable taking values in $R$, which is independent of $F_{\infty}^{B}$ with $E\left(\left|x_{0}\right|^{2}\right)<\infty . \alpha(\cdot)$ and $\beta(\cdot)$ are unknown drift parameters, while $\sigma(\cdot, \cdot)$ is an unknown diffusion coefficient.

Now we discuss the local stationary of model (1). We will approximate model (1) by a family of stationary processes indexed by $u \in[0,1]$. The stochastic process $\left\{\tilde{X}_{u, t}\right\}$ satisfies

$$
d \tilde{X}_{u, t}=\left[\alpha(u)+\beta(u) g\left(\tilde{X}_{u, t}\right)\right] d t+\sigma\left(u, \tilde{X}_{u, t}\right) d B_{t}, \quad \tilde{X}_{u, 0}=\tilde{x}_{u, 0},
$$

where $\tilde{x}_{u, 0}$ is a given random variable with $E\left(\left|\tilde{x}_{u, 0}\right|^{2}\right)<\infty$ for each $u \in[0,1]$. We shall require the following assumptions which ensure that model (2) has a family of stationary solutions (see Koo and Linton [3]).

(A1) The functions $\alpha(\cdot), \beta(\cdot), g(\cdot)$ and $\sigma(\cdot, \cdot)$ are twice boundedly continuously differentiable on $I$.

(A2) $\sigma^{2}(u, x)>0$ for each $x \in I$.

(A3) For all $x \in I$, there exists $\varepsilon>0$ such that $\int_{x-\varepsilon}^{x+\varepsilon} \frac{|[\alpha(u)+\beta(u) g(y)]|}{\sigma^{2}(u, y)} d y<\infty$.

(A4) The scale function defined as $S(u, x)=\int_{c}^{x} s(u, y) d y$, where $s(u, y)=\exp \left\{-2 \int_{c}^{y} \frac{\alpha(u)+\beta(u) g(z)}{\sigma^{2}(u, z)} d z\right\}$ for $x \in I$, satisfies the following: for a fixed number $c \in I$, we have $\int_{l}^{c} s(u, y) d y<\infty$ and $\int_{c}^{r} s(u, y) d y<\infty$.

(A5) The speed measure $\int_{l}^{r}\left[s(u, x) \sigma^{2}(u, x)\right]^{-1} d x<\infty$.

Under assumptions (A1)-(A5), for each time point $x \in I$, the process $\left\{\tilde{X}_{u, t}\right\}$ is strictly stationary and weekly dependent with a stationary density denoted by $f(u, \cdot)$, please refer to Karatzas and Shreve [6].

Definition 1 The process $\left\{X_{t, T}\right\}$ is locally stationary if for each rescaled time point $u \in$ $[0,1]$, there exists an associated process $\left\{\tilde{X}_{u, t}\right\}$ represented by (2) and satisfying assumptions (A1)-(A5) such that

$$
\left\|X_{t, T}-\tilde{X}_{u, t}\right\| \leq\left(\left|\frac{t}{T}-u\right|+\frac{1}{T}\right) U_{t, T}(u) \quad \text { a.s. }
$$

where $U_{t, T}(u)$ is a process of positive variables satisfying $E\left[\left(U_{t, T}(u)\right)^{\rho}\right]<C$ for some $\rho>0$ and $C<\infty$ independent of $u, t$ and $T .\|\cdot\|$ denotes an arbitrary norm on $R$.

From Definition 1, we have

$$
\left\|X_{t, T}-\tilde{X}_{u, t}\right\|=O_{p}\left(\left|\frac{t}{T}-u\right|+\frac{1}{T}\right) .
$$


Since equation (3) implies that in the neighborhood of a time point $u$, we can replace $X_{t, T}$ by $\tilde{X}_{u, t}$ for our asymptotic analysis, it allows us to approximate $X_{t, T}$ by a family of $\tilde{X}_{u, t}$ as $T \rightarrow \infty$.

Suppose that assumptions (A1)-(A5) hold, and $\beta(\cdot), \sigma(\cdot, \cdot)$ in model (1) satisfy

$$
\sup _{u \in[0,1]}|\beta(u)|<2 ; \quad \inf _{u \in[0,1]}|\beta(u)|>0 ; \quad \sup _{u \in[0,1], x \in I}|\sigma(u, x)|<1 .
$$

Then the diffusion process $\left\{X_{t, T}\right\}$ is locally stationary (see Theorem 1 of Koo and Linton [3]). Condition (4) is sufficient but need not be necessary.

\section{Local polynomial estimations of drift parameters}

In this section, we study the local polynomial estimations of time-varying drift parameters in model (1). Let the data $\left\{X_{t, T}, t=0,1,2, \ldots, T ; T=1,2, \ldots\right\}$ be sampled at discrete time points, and suppose that the time points are equally spaced. Denote $Y_{t, T}=X_{t+1, T}-X_{t, T}$, $\varepsilon_{t}=W_{t+1}-W_{t}$ and $\Delta=(t+1)-t=1$. The distance between two sampling points, $\Delta$, will not shrink but remains fixed. According to the independent increment property of Brownian motion $B_{t}$, the $\varepsilon_{t}$ is independent and normally distributed with mean zero and variance $\Delta$. Thus the discretized version of model (1) can be expressed as

$$
Y_{t, T}=\left[\alpha(t / T)+\beta(t / T) g\left(X_{t, T}\right)\right] \Delta+\sigma\left(t / T, X_{t, T}\right) \varepsilon_{t} .
$$

Based on the findings in Stanton [7] and Fan [8], the first-order discretized approximation error to the continuous-time model is extremely small, as long as data are sampled monthly or more frequently. Their findings simplify the estimation procedure.

Similarly, the discretized version of model (2) can be expressed as

$$
\tilde{Y}_{u, t}=\left[\alpha(u)+\beta(u) g\left(\tilde{X}_{u, t}\right)\right] \Delta+\sigma\left(u, \tilde{X}_{u, t}\right) \varepsilon_{t},
$$

where $\tilde{Y}_{u, t}=\tilde{X}_{u, t+1}-\tilde{X}_{u, t}$.

Based on equation (6), we discuss the local estimations of time-varying parameters $\alpha(u)$ and $\beta(u)$. By using $p$-polynomial fitting for $\alpha(u)$ and $\beta(u)$ (see Fan and Gijbels [5]), we can obtain the local estimations of time-varying parameters $\alpha(u)$ and $\beta(u)$ by minimizing the following objective function,

$$
\sum_{t=1}^{T}\left[\tilde{Y}_{u, t}-\left(\sum_{i=0}^{p} a_{i}(u-t / T)^{i}\right)-\left(\sum_{i=0}^{p} b_{i}(u-t / T)^{i}\right) g\left(\tilde{X}_{u, t}\right)\right]^{2} K_{h}(u-t / T),
$$

where $\left(a_{0}, b_{0}, a_{1}, \ldots, a_{p}, b_{1}, \ldots, b_{p}\right)^{T}$ is an unknown parameter vector, $K$ is a real-valued kernel function, $h$ is a bandwidth and $K_{h}(\cdot)=K(\cdot / h) / h$. Let $\tilde{\theta}(u)=\left(\tilde{a}_{0}, \tilde{b}_{0}, \tilde{a}_{1}, \ldots, \tilde{a}_{p}, \tilde{b}_{1}, \ldots, \tilde{b}_{p}\right)^{T}$ be the minimizer of locally weighted function $(7)$. Then

$$
\tilde{\theta}(u)=\left(\tilde{X}^{T} W \tilde{X}\right)^{-1} \tilde{X}^{T} W \tilde{Y},
$$

where $\tilde{Y}=\left(\tilde{Y}_{u, 1}, \tilde{Y}_{u, 2}, \ldots, \tilde{Y}_{u, T}\right)^{T}, W=\operatorname{diag}\left(K_{h}(u-t / T)\right)$ is a $T \times T$ diagonal matrix, and $\tilde{X}=\left(\tilde{Z}_{u, 1}, \tilde{Z}_{u, 2}, \ldots, \tilde{Z}_{u, T}\right)^{T}$, where $\tilde{Z}_{u, t}=\left(1, g\left(\tilde{X}_{u, t}\right), u-t / T, \ldots,(u-t / T)^{p}, g\left(\tilde{X}_{u, t}\right)(u-\right.$ $\left.t / T), \ldots, g\left(\tilde{X}_{u, t}\right)(u-t / T)^{p}\right)^{T}$. 
Let $\tilde{\alpha}(u)=\tilde{a}_{0}$ and $\tilde{\beta}(u)=\tilde{b}_{0}$. In matrixes $\tilde{X}$ and $\tilde{Y}$, we replace $\tilde{X}_{u, t}$ and $\tilde{Y}_{u, t}$ by $X_{t, T}$ and $Y_{t, T}=X_{t+\Delta, T}-X_{t, T}$, respectively. Then we can obtain the matrixes based on sample data, denoting $X$ and $Y$, respectively. Let

$$
\hat{\theta}(u)=\left(\hat{a}_{0}, \hat{b}_{0}, \hat{a}_{1}, \ldots, \hat{a}_{p}, \hat{b}_{1}, \ldots, \hat{b}_{p}\right)^{T}=\left(X^{T} W X\right)^{-1} X^{T} W Y .
$$

Then the local polynomial estimations of $\alpha(u)$ and $\beta(u)$ are

$$
\hat{a}(u)=\hat{a}_{0} \quad \text { and } \quad \hat{b}(u)=\hat{b}_{0},
$$

respectively. Due to equation (3), $\tilde{\theta}(u)$ and $\hat{\theta}(u)$ share the same asymptotic properties, and so do $\tilde{\alpha}(u)$ and $\hat{\alpha}(u), \tilde{\beta}(u)$ and $\hat{\beta}(u)$, respectively.

Now we establish the asymptotic properties of the local polynomial estimations of the drift parameters as the following. We first give regularity conditions for model (1). Denote

$$
\hat{f}(u, x)=\frac{1}{T h_{1} h_{2}} \sum_{t=1}^{T} K_{h_{1}}(u-t / T) K_{h_{2}}\left(x-X_{t, T}\right) .
$$

This can be interpreted as an estimation of $f(u, x)$.

(C1) The diffusion process $\left\{X_{t, T}\right\}$ is a locally stationary process represented by (2).

(C2) For all $x \in I$ and $u \in[0,1], \alpha(u), \beta(u)$ and $\sigma^{2}(u, x)$ are twice continuously differentiable with $\sigma^{2}(u, x)>0$ and $\sup _{u \in[0,1]} \beta(u)<0$.

(C3) The kernel function $K(\cdot)$ is a bounded symmetric around zero Lipschitz continuous function with

$$
\begin{array}{ll}
\int|K(z)| d z<\infty, & \int K(z) d z=1, \\
\int|z|^{r}|K(z)| d z<\infty & (r \geq 2) .
\end{array}
$$

(C4) Bandwidths $h, h_{1}$ and $h_{2}$ satisfy: as $T \rightarrow \infty, h \rightarrow 0$ and $T h \rightarrow \infty$; $\max \left\{h_{1}, h_{2}\right\} \rightarrow 0$ and $T h_{1} h_{2} \rightarrow \infty ; h / h_{1} h_{2} \rightarrow \infty$.

(C5) For $t_{1}<t_{2}<t_{3}<t_{4}$, the density $f_{u, t_{1}, t_{2}, t_{3}, t_{4}}$ of $\left(\tilde{X}_{u, t_{1}}, \tilde{X}_{u, t_{2}}, \tilde{X}_{u, t_{3}}, \tilde{X}_{u, t_{4}}\right)^{T}$ exists with $\sup _{t_{1}<t_{2}<t_{3}<t_{4}}\left\|f_{u, t_{1}, t_{2}, t_{3}, t_{4}}\right\|_{\infty}<\infty$.

(C6) For some positive $\eta$, $\sup _{T} E\left[\left|X_{t, T}\right|^{4+\eta}\right]<\infty$.

The following Theorem 1 and Theorem 2 show the weak consistency and asymptotic normality of our proposed local estimations, respectively.

Theorem 1 Suppose that assumptions (C1)-(C6) hold. Let $u$ be an interior point over $[0,1]$. Then, as $T \rightarrow \infty$, we have

$$
\hat{\alpha}(u) \rightarrow_{P} \alpha(u), \quad \hat{\beta}(u) \rightarrow_{P} \beta(u),
$$

where $\rightarrow_{P}$ means convergence in probability. 
Theorem 2 Suppose that assumptions $(\mathrm{C} 1)-(\mathrm{C} 6)$ hold. Let $u$ be an interior point over $[0,1]$. Then, as $T \rightarrow \infty$, we have

$$
\sqrt{T h}\left[\left(\begin{array}{c}
\hat{\alpha}(u) \\
\hat{\beta}(u)
\end{array}\right)-\left(\begin{array}{c}
\alpha(u) \\
\beta(u)
\end{array}\right)-h^{2} \operatorname{bias}_{\alpha \beta}(u)\right] \rightarrow{ }_{d} N\left(0, V(u)_{\alpha \beta}\right),
$$

where $\rightarrow_{d}$ means convergence in distribution, $\operatorname{bias}_{\alpha \beta}(u)=\mu_{2}(K) \Phi_{u}$ and $V(u)_{\alpha \beta}=\|K\|_{2}^{2} \Sigma_{u}$, where $\mu_{2}(K)=\int z^{2} K(z) d z, \Phi_{u}$ is a $2 \times 1$ vector whose elements are the first two elements of vector $\Psi_{u}$, where

$$
\Psi_{u}=M_{u}^{-1} E\left[\tilde{Z}_{u, t} \tilde{Z}_{u, t}^{T}\left\{\theta^{\prime}(u) \frac{\partial \ln f\left(u, \tilde{X}_{u, t}\right)}{\partial u}+\frac{1}{2} \theta^{\prime \prime}(u)\right\}\right]
$$

$\Sigma_{u}=\left[M_{u}^{-1} \Omega_{u} M_{u}^{-1}\right]_{22}$, where $M_{u}=E\left[\tilde{Z}_{u, t} \tilde{Z}_{u, t}^{T}\right], \Omega_{u}=E\left[\tilde{Z}_{u, t} \tilde{Z}_{u, t}^{T} \sigma^{2}\left(u, \tilde{X}_{u, t}\right)\right],[\cdot]_{22}$ denotes the upper-left $2 \times 2$ sub-matrix.

In order to discuss the uniform convergence of our proposed estimations, we further give the following two assumptions.

(C7) The diffusion process $\left\{X_{t, T}\right\}$ is strongly mixing with its mixing coefficients $\alpha(K)$ such that for $\rho>0$,

$$
\alpha(K)=\sup _{-T \leq t \leq T} \sup _{A \in F_{-\infty, B \in F_{T, t+k}^{T, t}}^{\infty}}|P(A B)-P(A) P(B)|
$$

where $\alpha(K)$ converges exponentially fast to zero as $k \rightarrow \infty$.

(C8) The density $g_{t}(x)$ of $\left\{X_{t, T}\right\}$ and joint densities $g_{t, t+j}(x, y)$ of $\left(X_{t, T}, X_{t+j, T}\right)^{T}$ are uniformly bounded.

Assumptions (C7) and (C8) allow us to make use of the asymptotic independence property for heterogeneous data.

Theorem 3 Suppose that assumptions (C1)-(C8) hold and $\sqrt{\frac{\ln T}{T h}}=o(1)$. Then, for a compact set $I$ and any sequence $\delta_{T}$ such that $\delta_{T} / h_{1} \rightarrow \infty$ and $\delta_{T} \rightarrow 0$, we have

$$
\begin{aligned}
& \sup _{u \in\left[\delta_{T}, 1-\delta_{T}\right]}|\hat{\alpha}(u)-\alpha(u)|=O_{P}\left(h^{2}\right)+O_{P}\left(\sqrt{\frac{\ln T}{T h}}\right), \\
& \sup _{u \in\left[\delta_{T}, 1-\delta_{T}\right]}|\hat{\beta}(u)-\beta(u)|=O_{P}\left(h^{2}\right)+O_{P}\left(\sqrt{\frac{\ln T}{T h}}\right) .
\end{aligned}
$$

The proofs of Theorem 1, Theorem 2 and Theorem 3 are given in Section 5.

\section{The estimation of the diffusion coefficient}

In this section, we study the estimation of the diffusion coefficient. From the forward Kolmogorov equation (see (5.1.6) in Karatzas and Shreve [6]), we have

$$
\sigma^{2}(u, x)=\frac{2}{f(u, x)} \int_{-\infty}^{x}[\alpha(u)+\beta(u) g(y)] f(u, y) d y,
$$


where $f(u, x)$ is the density of $\tilde{X}_{u, t}$. Since we already have estimations of $\alpha(u), \beta(u)$ and $f(u, x)$, we can use these to estimate $\sigma^{2}(u, x)$ as follows,

$$
\hat{\sigma}^{2}(u, x)=\frac{2}{\hat{f}(u, x)} \int_{-\infty}^{x}[\hat{\alpha}(u)+\hat{\beta}(u) g(y)] \hat{f}(u, y) d y .
$$

The estimation in (14) is computed by numerical one-dimensional integration.

Theorem 4 Suppose that assumptions (C1)-(C6) hold. Let $u$ be an interior point over $[0,1]$. Then, as $T \rightarrow \infty$, we have

$$
\hat{\sigma}^{2}(u, x) \rightarrow_{P} \sigma^{2}(u, x)
$$

and

$$
\sqrt{T h_{1} h_{2}}\left(\hat{\sigma}^{2}(u, x)-\sigma^{2}(u, x)-\operatorname{bias}_{\sigma}(u)\right) \rightarrow_{d} N\left(0, V_{\sigma}(u, x)\right),
$$

where $\operatorname{bias}_{\sigma}(u)=\frac{\mu_{2}(K)}{2 f(u, x)} \sigma^{2}(u, x)\left[h_{1} \frac{\partial^{2} f(u, x)}{\partial u^{2}}+h_{2} \frac{\partial^{2} f(u, x)}{\partial x^{2}}\right], V_{\sigma}(u, x)=\|K\|_{2}^{4} \frac{\sigma^{4}(u, x)}{f(u, x)}$.

Theorem 5 Suppose that assumptions (C1)-(C8) hold and $\sqrt{\frac{\ln T}{T h_{1} h_{2}}}=o(1)$. Then, for a compact set $I$ and any sequence $\delta_{T}$ such that $\delta_{T} / h_{1} \rightarrow \infty$ and $\delta_{T} \rightarrow 0$, we have

$$
\sup _{u \in\left[\delta_{T}, 1-\delta_{T}\right]}\left|\hat{\sigma}^{2}(u, x)-\sigma^{2}(u, x)\right|=O_{P}\left(h^{2}\right)+O_{P}\left(\sqrt{\frac{\ln T}{T h_{1} h_{2}}}\right) .
$$

Theorem 4 shows the consistence and asymptotic normality of the estimation of the diffusion coefficient, and Theorem 5 gives the uniform convergence rate of our estimation.

\section{Proofs of main results}

Denote

$$
Z_{t, T}=\left(\left(1, g\left(X_{t, T}\right), u-t / T, \ldots,(u-t / T)^{p}, g\left(X_{t, T}\right)(u-t / T), \ldots, g\left(X_{t, T}\right)\right)(u-t / T)^{p}\right)^{T} .
$$

Proof of Theorem 1 Due to equations (8) and (9), we have

$$
\begin{aligned}
& \hat{\theta}(u)=\left[\sum_{t=1}^{T} K_{u t} Z_{t, T} Z_{t, T}^{T}\right]^{-1}\left[\sum_{t=1}^{T} K_{u t} Z_{t, T} Y_{t, T}\right], \\
& \tilde{\theta}(u)=\left[\sum_{t=1}^{T} K_{u t} \tilde{Z}_{u t} \tilde{Z}_{u t}^{T}\right]^{-1}\left[\sum_{t=1}^{T} K_{u t} \tilde{Z}_{u t} \tilde{Y}_{u t}\right] .
\end{aligned}
$$

By using equation (3) and $\left\{X_{t, T}\right\}$ is strongly mixing, in the neighborhood of $u$, we have

$$
\begin{aligned}
& \frac{1}{T} \sum_{t=1}^{T} K_{u t} Z_{t, T} Z_{t, T}^{T} \rightarrow_{P} E\left[\tilde{Z}_{u t} \tilde{Z}_{u t}^{T}\right], \\
& \frac{1}{T} \sum_{t=1}^{T} K_{u t} \tilde{Z}_{u t} \tilde{Z}_{u t}^{T} \rightarrow_{P} E\left[\tilde{Z}_{u t} \tilde{Z}_{u t}^{T}\right] .
\end{aligned}
$$


Let $M_{u} \doteq E\left[\tilde{Z}_{u t} \tilde{Z}_{u t}^{T}\right] . M_{u}$ denotes expectation of $\tilde{Z}_{u t} \tilde{Z}_{u t}^{T}$ in the vicinity of the fixed time $u$. Due to Lemma A.5 in Dahlhaus and Subba Rao [9] and Lemma 2 of A.1 in Fryzlewicz et al. [10], we have

$$
\begin{aligned}
& \frac{1}{T} \sum_{t=1}^{T} K_{u t} Z_{t, T} Y_{t, T} \rightarrow_{P} E\left[\tilde{Z}_{u t} \tilde{Y}_{u t}\right], \\
& \frac{1}{T} \sum_{t=1}^{T} K_{u t} \tilde{Z}_{u t} \tilde{Y}_{u t} \rightarrow_{P} E\left[\tilde{Z}_{u t} \tilde{Y}_{u t}\right] .
\end{aligned}
$$

Let $\tilde{Y}_{u t}-\tilde{Z}_{u t}^{T} \theta(t / T)=v_{u t}$, where $\theta(u)=\left(\alpha(u), \beta(u), \alpha^{\prime}(u), \ldots, \alpha^{(p)} 1 / p !, \beta^{\prime}(u), \ldots, \beta^{(p)} 1 / p !\right)^{T}$. Then

$$
\begin{aligned}
\tilde{\theta}(u)-\theta(u)= & {\left[\sum_{t=1}^{T} K_{u t} \tilde{Z}_{u t} \tilde{Z}_{u t}^{T}\right]^{-1}\left[\sum_{t=1}^{T} K_{u t} \tilde{Z}_{u t} \tilde{Y}_{u t}\right]-\theta(u) } \\
= & {\left[\sum_{t=1}^{T} K_{u t} \tilde{Z}_{u t} \tilde{Z}_{u t}^{T}\right]^{-1} \sum_{t=1}^{T} K_{u t} \tilde{Z}_{u t}\left[\tilde{Z}_{u t}^{T} \theta(t / T)+v_{u t}\right]-\theta(u) } \\
= & {\left[\sum_{t=1}^{T} K_{u t} \tilde{Z}_{u t} \tilde{Z}_{u t}^{T}\right]^{-1} \sum_{t=1}^{T} K_{u t} \tilde{Z}_{u t} \tilde{Z}_{u t}^{T}[\theta(t / T)-\theta(u)] } \\
& +\left[\sum_{t=1}^{T} K_{u t} \tilde{Z}_{u t} \tilde{Z}_{u t}^{T}\right]^{-1} \sum_{t=1}^{T} K_{u t} \tilde{Z}_{u t} v_{u t} \\
\doteq & {\left[M_{T}(u)\right]^{-1}\left[I_{1 T}(u)+I_{2 T}(u)\right], }
\end{aligned}
$$

where $M_{T}(u)=\sum_{t=1}^{T} K_{u t} \tilde{Z}_{u t} \tilde{Z}_{u t}^{T}, I_{1 T}(u)=\sum_{t=1}^{T} K_{u t} \tilde{Z}_{u t} \tilde{Z}_{u t}^{T} \theta(t / T)-\theta(u), I_{2 T}(u)=\sum_{t=1}^{T} K_{u t} \times$ $\tilde{Z}_{u t} v_{u t}$. For $B>0$, we have

$$
\begin{aligned}
E\left[\left\|\frac{1}{T} I_{1 T}(u)\right\|\right] \leq & \sup _{|T u-t| \leq B h}\|\tilde{\theta}(t / T)-\theta(u)\| \frac{1}{T} \sum_{|T u-t| \leq B T h}\left|K_{u t}\right| \operatorname{tr}\left(M_{u}\right) \\
& +\frac{C}{T} \sum_{|T u-t|>B T h}\left|K_{u t}\right| \operatorname{tr}\left(M_{u}\right) \\
\leq & C\left[\sup _{|T u-t| \leq B h}\|\tilde{\theta}(t / T)-\theta(u)\|+\int_{|u| \geq B}|K(u)| d u\right]+o(1) .
\end{aligned}
$$

Finally,

$$
E\left[\left\|\frac{1}{T} I_{2 T}(u)\right\|\right]=\frac{1}{T^{2}} \sum_{t=1}^{T} K_{u t}^{2} \operatorname{tr}\left(M_{u}\right) \sigma^{2}=O_{P}\left(\frac{1}{T h}\right) .
$$

Therefore, $\tilde{\theta}(u) \rightarrow_{P} \theta(u)$, which implies $\hat{\theta}(u) \rightarrow_{p} \theta(u)$. This completes the proof of Theorem 1.

Proof of Theorem 2 We start with the asymptotic normality of $\tilde{\theta}(u)$. Continuing from equation (18), we have

$$
\tilde{\theta}(u)=\left[M_{T}(u)\right]^{-1}\left[I_{1 T}(u)+I_{2 T}(u)\right]+\theta(u) .
$$


We have proved that $M_{T}(u)=M_{u}+o(1)$ in the proof of Theorem 1. We discuss $E\left[I_{1 T}(u)\right]$ in the following and we have

$$
\begin{aligned}
E\left[I_{1 T}(u)\right] & =E\left[\sum_{t=1}^{T} K_{u t} \tilde{Z}_{u t} \tilde{Z}_{u t}^{T}(\theta(t / T)-\theta(u))\right] \\
& =\iint z_{1} z_{1}^{T}(\theta(u+h v)-\theta(u)) f(u+h v, x) K(v) d x d v \\
& =\iint z_{1} z_{1}^{T}\left[h v \theta^{\prime}(u)+1 / 2 h^{2} v^{2} \theta^{\prime \prime}(u)\right]\left[f(u, x)+\frac{\partial f(u, x)}{\partial u} h v\right] K(v) d x d v \\
& =\iint z_{1} z_{1}^{T}\left[\frac{\partial f(u, x)}{\partial u} h^{2} v^{2} \theta^{\prime}(u)+1 / 2 f(u, x) h^{2} v^{2} \theta^{\prime \prime}(u)\right] K(v) d x d v+o\left(h^{2}\right) \\
& =h^{2} \int v^{2} K(v) d v \int z_{1} z_{1}^{T}\left[\frac{\partial f(u, x)}{\partial u} \theta^{\prime}(u)+1 / 2 \theta^{\prime \prime}(u)\right] f(u, x) d x+o\left(h^{2}\right) \\
& =h^{2}\left[\mu_{2}(K)\right] E_{u}\left[z_{1} z_{1}^{T}\left\{\frac{\partial f(u, x)}{\partial u} \theta^{\prime}(u)+1 / 2 \theta^{\prime \prime}(u)\right\}\right]+o\left(h^{2}\right) .
\end{aligned}
$$

Note that $\left\{v_{u t}\right\}$ is a martingale difference sequence. Then

$$
\sqrt{T h} I_{2 T}(u) \rightarrow{ }_{d} N\left(0,\|K\|_{2}^{2} \Omega_{u}\right)
$$

where $\Omega_{u}=E_{u}\left[z_{1} z_{1}^{T} \sigma^{2}\left(u, \tilde{X}_{u, t}\right)\right]$. By using $\hat{\theta}(u)$ and $\tilde{\theta}(u)$ having the same asymptotic property, we have

$$
\sqrt{T h}\left\{\hat{\theta}(u)-\theta(u)-h^{2} \mu_{2}(K) \Psi_{u}\right\} \rightarrow_{d} N\left(0,\|K\|_{2}^{2} M_{u}^{-1} \Omega_{u} M_{u}^{-1}\right) .
$$

This completes the proof of Theorem 2 .

Proof of Theorem 3 Let $g_{t, T}(x)$ be the density of $X_{t, T}$. Denote

$$
\begin{aligned}
& H_{0}=\sup _{t, T \geq 1} \sup _{x \in R} g_{t, T}(x), \\
& H_{Y, 1}=\sup _{t, T \geq 1} \sup _{x \in R} E\left[Y_{t, T} \mid X_{t, T}=x\right] g_{t, T}(x), \\
& H_{Y, 2}=\sup _{T \geq 1|t-j| \geq M} \sup _{x \in R} E\left[Y_{t, T} Y_{j, T} \mid X_{t, T}=x, X_{j, T}=y\right] g_{t, j, T}(x, y),
\end{aligned}
$$

where $M$ is some positive number. Under assumptions (C1)-(C8), conditions for Theorem 1 in Kristensen [11] are met and application of Theorem 1 can be used.

Let us start with $\hat{\beta}(u)$. Let

$$
\begin{aligned}
& \hat{Q}_{1}=\left[\frac{1}{T} \sum_{t=1}^{T} K_{u t}\right]\left[\frac{1}{T} \sum_{t=1}^{T} K_{u t} X_{t, T}^{2}\right]-\left[\frac{1}{T} \sum_{t=1}^{T} K_{u t} X_{t, T}\right]^{2}, \\
& \hat{Q}_{2}=\frac{1}{T} \sum_{t=1}^{T} K_{u t} X_{t, T} Y_{t, T}-\left[\frac{1}{T} \sum_{t=1}^{T} K_{u t} X_{t, T}\right]\left[\frac{1}{T} \sum_{t=1}^{T} K_{u t} Y_{t, T}\right] .
\end{aligned}
$$


Note that $H_{0}, H_{Y, 1}$ and $H_{Y, 2}$ are local demeaned values. According to Theorem 1 in Kristensen [11], we have

$$
\begin{aligned}
& \sup _{u \in\left[\delta_{T}, 1-\delta_{T}\right]}\left|\hat{Q}_{1}-E\left[\hat{Q}_{1}\right]\right|=O_{P}\left(\sqrt{\frac{\ln T}{T h}}\right), \\
& \sup _{u \in\left[\delta_{T}, 1-\delta_{T}\right]}\left|\hat{Q}_{2}-E\left[\hat{Q}_{2}\right]\right|=O_{P}\left(\sqrt{\frac{\ln T}{T h}}\right) .
\end{aligned}
$$

Also, due to standard nonparametric estimation manipulation, we obtain

$$
\begin{aligned}
& \sup _{u \in\left[\delta_{T}, 1-\delta_{T}\right]}\left|E\left[\hat{Q}_{1}-Q_{1}\right]\right|=O\left(h^{2}\right), \\
& \sup _{u \in\left[\delta_{T}, 1-\delta_{T}\right]}\left|E\left[\hat{Q}_{2}-Q_{2}\right]\right|=O\left(h^{2}\right) .
\end{aligned}
$$

Therefore,

$$
\begin{aligned}
& \sup _{u \in\left[\delta_{T}, 1-\delta_{T}\right]}\left|\hat{Q}_{1}-Q_{1}\right|=O\left(h^{2}\right)+O_{P}\left(\sqrt{\frac{\ln T}{T h}}\right), \\
& \sup _{u \in\left[\delta_{T}, 1-\delta_{T}\right]}\left|\hat{Q}_{2}-Q_{2}\right|=O\left(h^{2}\right)+O_{P}\left(\sqrt{\frac{\ln T}{T h}}\right) .
\end{aligned}
$$

By using the Taylor expansion, we have

$$
|\hat{\beta}(u)-\beta(u)|=\left|\frac{\hat{Q}_{2}}{\hat{Q}_{1}}-\beta(u)\right|=\left|\frac{\hat{Q}_{2}}{\hat{Q}_{1}}-\frac{Q_{2}}{Q_{1}}\right| \leq \frac{\left|\hat{Q}_{2}-Q_{2}\right|}{Q_{1}}+\frac{\left|Q_{2}\right|}{Q_{1}^{2}}\left|\hat{Q}_{1}-Q_{1}\right| .
$$

Due to the same argument as in Kristensen [11], the uniform convergence rate of the above equation is determined by $\left|\hat{Q}_{1}-Q_{1}\right|$ and $\left|\hat{Q}_{2}-Q_{2}\right|$. Therefore,

$$
\sup _{u \in\left[\delta_{T}, 1-\delta_{T}\right]}|\hat{\beta}(u)-\beta(u)|=O\left(h^{2}\right)+O_{P}\left(\sqrt{\frac{\ln T}{T h}}\right) .
$$

As for $\hat{\alpha}(u)$, the method in Hansen [12] applies with the previous result for $\hat{\beta}(u)$ and the rate of uniform convergence of $\hat{\alpha}(u)$ is determined by that of $\hat{\beta}(u)$. Therefore,

$$
\sup _{u \in\left[\delta_{T}, 1-\delta_{T}\right]}|\hat{\alpha}(u)-\alpha(u)|=O\left(h^{2}\right)+O_{P}\left(\sqrt{\frac{\ln T}{T h}}\right) .
$$

This completes the proof of Theorem 3.

Proof of Theorem 4 We first discuss the consistency of our estimation. Recall that

$$
\begin{aligned}
\hat{\sigma}^{2}(u, x)-\sigma^{2}(u, x)= & \frac{2}{\hat{f}(u, x)} \int_{-\infty}^{x}[\hat{\alpha}(u)+\hat{\beta}(u) g(y)] \hat{f}(u, y) d y \\
& -\frac{2}{f(u, x)} \int_{-\infty}^{x}[\alpha(u)+\beta(u) g(y)] f(u, y) d y
\end{aligned}
$$




$$
\begin{aligned}
= & \frac{2}{\hat{f}(u, x)} \int_{-\infty}^{x}[\alpha(u)+\beta(u) g(y)] f(u, y) d y \\
& -\frac{2}{\hat{f}(u, x)} \int_{-\infty}^{x}[\alpha(u)+\beta(u) g(y)] f(u, y) d y \\
& +\frac{2}{\hat{f}(u, x)} \int_{-\infty}^{x}[\hat{\alpha}(u)+\hat{\beta}(u) g(y)] \hat{f}(u, y) d y \\
& -\frac{2}{f(u, x)} \int_{-\infty}^{x}[\alpha(u)+\beta(u) g(y)] f(u, y) d y \\
= & {\left[\frac{1}{\hat{f}(u, x)}-\frac{1}{f(u, x)}\right] 2 \int_{-\infty}^{x}[\alpha(u)+\beta(u) g(y)] f(u, y) d y } \\
& +\frac{2}{\hat{f}(u, x)}\left\{\int_{-\infty}^{x}[\hat{\alpha}(u)+\hat{\beta}(u) g(y)] \hat{f}(u, y) d y\right. \\
& \left.-\int_{-\infty}^{x}[\alpha(u)+\beta(u) g(y)] f(u, y) d y\right\} \\
= & I_{1}+I_{2},
\end{aligned}
$$

where

$$
I_{1}=\left[\frac{1}{\hat{f}(u, x)}-\frac{1}{f(u, x)}\right] 2 \int_{-\infty}^{x}[\alpha(u)+\beta(u) g(y)] f(u, y) d y
$$

and

$$
I_{2}=\frac{2}{\hat{f}(u, x)}\left\{\int_{-\infty}^{x}[\hat{\alpha}(u)+\hat{\beta}(u) g(y)] \hat{f}(u, y) d y-\int_{-\infty}^{x}[\alpha(u)+\beta(u) g(y)] f(u, y) d y\right\} .
$$

Let $H=\left[\frac{1}{\hat{f}(u, x)}-\frac{1}{f(u, x)}\right]$. According to Koo and Linton [3], we have that $f(u, x)$ is bounded away from zero and $\hat{f}(u, x)-f(u, x) \rightarrow_{P} 0$. Thus

$$
-\frac{1}{\hat{f}(u, x) f(u, x)}(\hat{f}(u, x)-f(u, x))+o_{P}(\|\hat{f}(u, x)-f(u, x)\|) \rightarrow_{P} 0,
$$

which implies that $\left|I_{1}\right| \rightarrow_{P} 0$. On the other hand, it can be easily shown that $\left|I_{2}\right| \rightarrow_{P} 0$ given the consistency of the marginal density and the drift shown before.

We can prove the asymptotic normality of $\sigma^{2}(u, x)$ by using a similar method of Theorem 3 in Koo and Linton [3]. Here, we do not give the details. This completes the proof of Theorem 4.

Proof of Theorem 5 Notice that the uniform convergence of the proposed estimation for the diffusion coefficient is determined by that of the estimation of the density function. Therefore, due to Lemma 6 in Koo and Linton [3], the proof of Theorem 5 is completed.

\section{Conclusions}

In this paper, the local polynomial estimations of time-varying drift parametric functions are proposed. By applying the forward Kolmogorov equation, the estimation of the diffusion coefficient is obtained. The consistency, asymptotic normality and uniform convergence of the proposed estimations are proved. 


\section{Competing interests}

The authors declare that they have no competing interests.

\section{Authors' contributions}

All authors contributed equally to the manuscript, read and approved the final manuscript.

\section{Acknowledgements}

This work is supported by the National Natural Science Foundation of China (No. 11171221) and Shanghai Leading Academic Discipline Project (No. XTKX2012). The authors would like to thank the anonymous referees for several useful interesting comments and suggestions about the paper.

Received: 20 October 2013 Accepted: 3 December 2013 Published: 14 Jan 2014

\section{References}

1. Dahlhaus, R: On the Kullback-Leibler information divergence of locally stationary processes. Stoch. Process. Appl. 62, 139-168 (1996)

2. Dahlhaus, R: Fitting time series models to nonstationary processes. Ann. Stat. 25, 1-37 (1997)

3. Koo, B, Linton, O: Semiparametric estimation of locally stationary diffusion models. J. Econom. 170, $210-233$ (2012)

4. Vogt, M: Nonparametric regression for locally stationary time series. Ann. Stat. 40, 2601-2633 (2012)

5. Fan, J, Gijbels, I: Local Polynomial Modelling and Its Applications. Chapman \& Hall, London (1996)

6. Karatzas, I, Shreve, S: Brownian Motion and Stochastic Calculus. Springer, New York (2000)

7. Stanton, R: A nonparametric model of term structure dynamics and the market price of interest rate risk. J. Finance 52, 1973-2002 (1997)

8. Fan, J, Zhang, C: A re-examination of Stanton's diffusion estimations with applications to financial model validation. J. Am. Stat. Assoc. 98, 118-134 (2003)

9. Dahlhaus, R, Subba Rao, S: Statistical inference for time varying arch processes. Ann. Stat. 34, 1075-1114 (2006)

10. Fryzlewicz, P, Sapatinas, T, Subba Rao, S: Normalised least squares estimation in time-varying ARCH models. Ann. Stat. 36, 742-786 (2008)

11. Kristensen, D: Uniform convergence rates of kernel estimations with heterogeneous dependent data. Econom Theory 25, 1433-1445 (2009)

12. Hansen, B: Uniform convergence rates for kernel estimations with dependent data. Econom. Theory 24, 726-748 (2008)

10.1186/1029-242X-2014-17

Cite this article as: Wang and Xiao: Local polynomial estimations of time-varying coefficients for local stationary diffusion models. Journal of Inequalities and Applications 2014, 2014:17

\section{Submit your manuscript to a SpringerOpen ${ }^{\ominus}$ journal and benefit from:}

- Convenient online submission

- Rigorous peer review

Immediate publication on acceptance

- Open access: articles freely available online

- High visibility within the field

- Retaining the copyright to your article 